\title{
Correction to: Acoustic Contrast Between Neutral and Angry Speech: Variation of Prosodic Features in Algerian Dialect Speech and German Speech
}

\author{
F. Ykhlef and D. Bouchaffra
}

\section{Correction to:}

Chapter 4 in: A. Khelassi, V. V. Estrela (eds.), Advances in Multidisciplinary Medical

Technologies - Engineering, Modeling and Findings, https://doi.org/10.1007/978-3-030-57552-6_4

Reference [18] is now corrected as follows:

F. Ykhlef, A. Derbal, W. Benzaba, R. Boutaleb, D. Bouchaffra, H. Meraoubi, Far. Ykhlef, in The IEEE International Conference on Advanced Electrical Engineering. Towards building an emotional speech corpus of Algerian dialect: criteria and preliminary assessment results. (Algiers, 2019). 